\title{
UPLC/MS/MS-Based Metabolomics Study of the Hepatotoxicity and Nephrotoxicity in Rats Induced by
}

\section{Polygonum multiflorum Thunb}

\author{
Yan Yan ${ }^{1 \#, ~ N i n g ~ S h i " ~}{ }^{2 \#}$ Xuyang $\mathrm{Han}^{3}$, Guodong $\mathrm{Li}^{4}$, Binyu Wen ${ }^{1 *}$, Jian Gao ${ }^{1,4 *}$
}

1. Dongfang Hospital, Beijing University of Chinese Medicine, Beijing 100078, P.R. China

2. Pharmaceutical Department of Characteristic Medical Center, Strategic Support Force, Beijing 100101, P.R. China

3. Beijing Institute of Traditional Chinese Medicine, Beijing Hospital of Traditional Chinese Medicine, Capital Medical University, Beijing 100010, P.R. China

4. Beijing University of Chinese Medicine, Beijing 100078, P.R. China

\#These authors contributed equally to this work.

*Correspondence:

Binyu Wen

E-mail: wen-binyu@163.com

Dongfang Hospital, Beijing University of Chinese Medicine

No. 6 Fangxingyuan 1st Block, Fengtai District, Beijing, 100078, PR. China.

Jian Gao

Beijing University of Chinese Medicine

No. 11, Bei San Huan Dong Lu, Chaoyang District, Beijing 100029, China

E-mail: gaojian_5643@163.com 


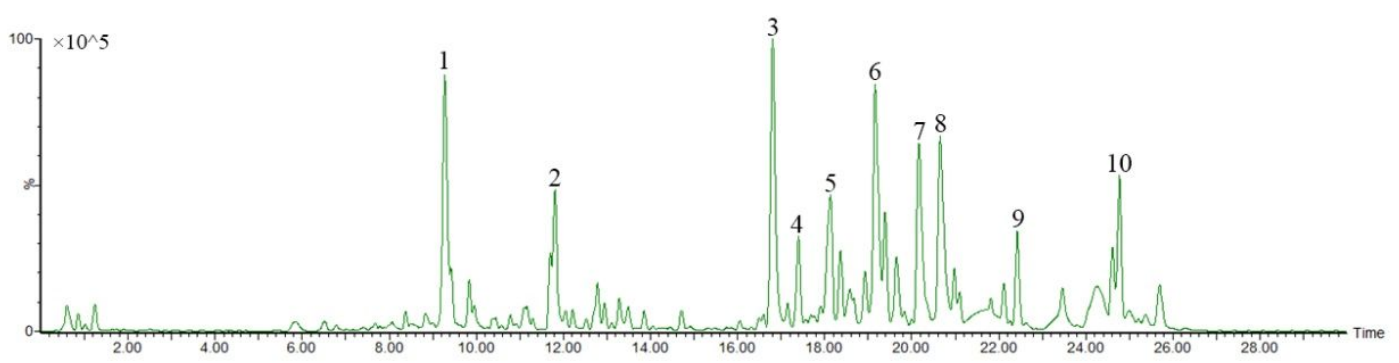

Figure S1 UPLC-Q-TOF-MS characteristic chromatogram of decoction of PM.

1. 2,3,5,4'-Tetrahydroxystilbene-2-O-glc; 2. Emodin-8-O-glucoside; 3.Emodin; 4.

Torachrysone-O-glucogallin; 5.Tetrahydroxystilbene-O-(galloyl)-hex; 6.

Emodin-O-hex-sulphate;7. Torachrysone-O-hex; 8. Emodin-O-(malonyl)-hex; 9. Emodin-O-(acetyl)-hex; 10. Trihydroxy-octadecenoic acid.
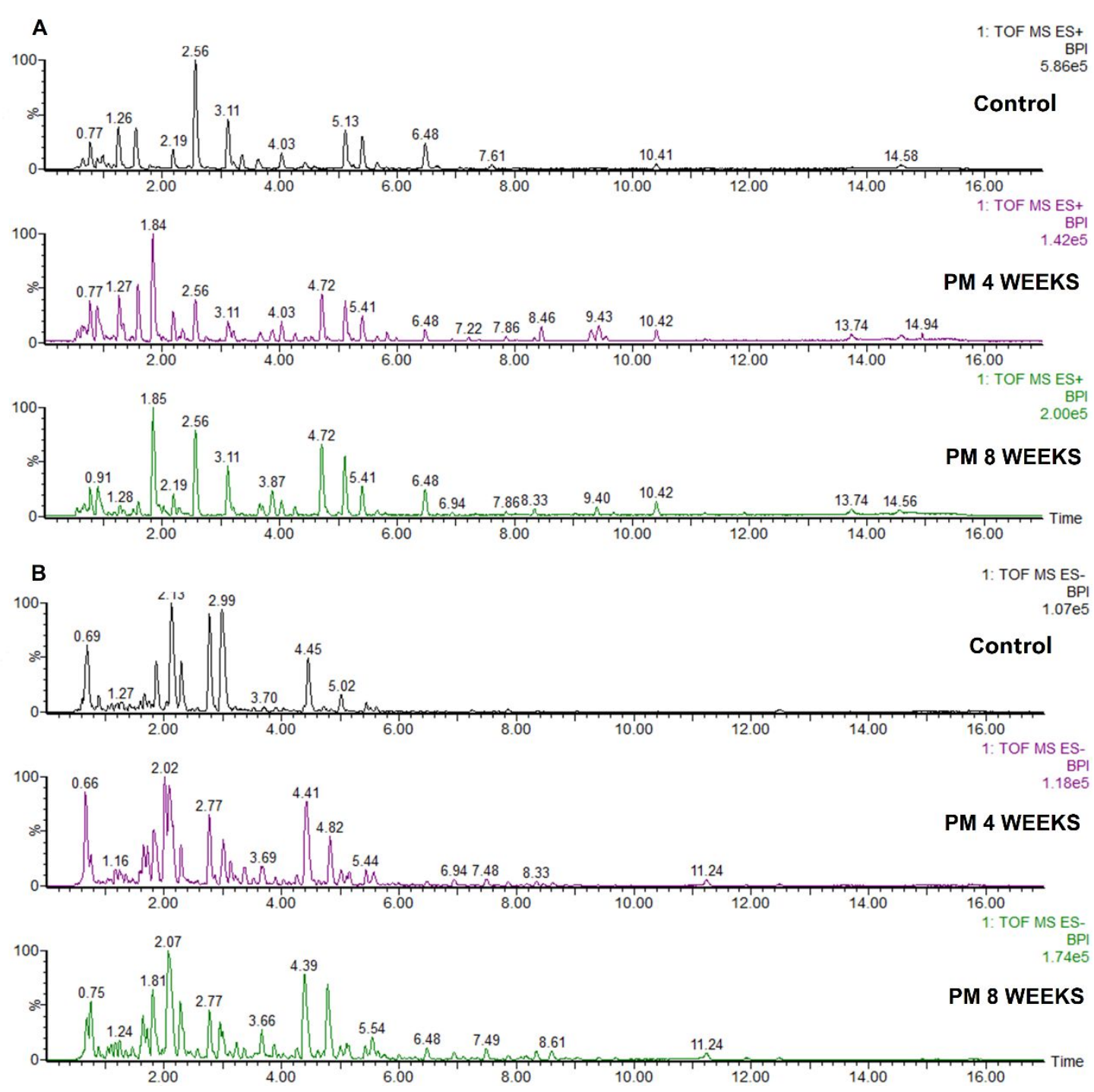

Figure S2. Representative BPI chromatograms from the positive and negative ions in urine samples (A-positive, B-negative). 


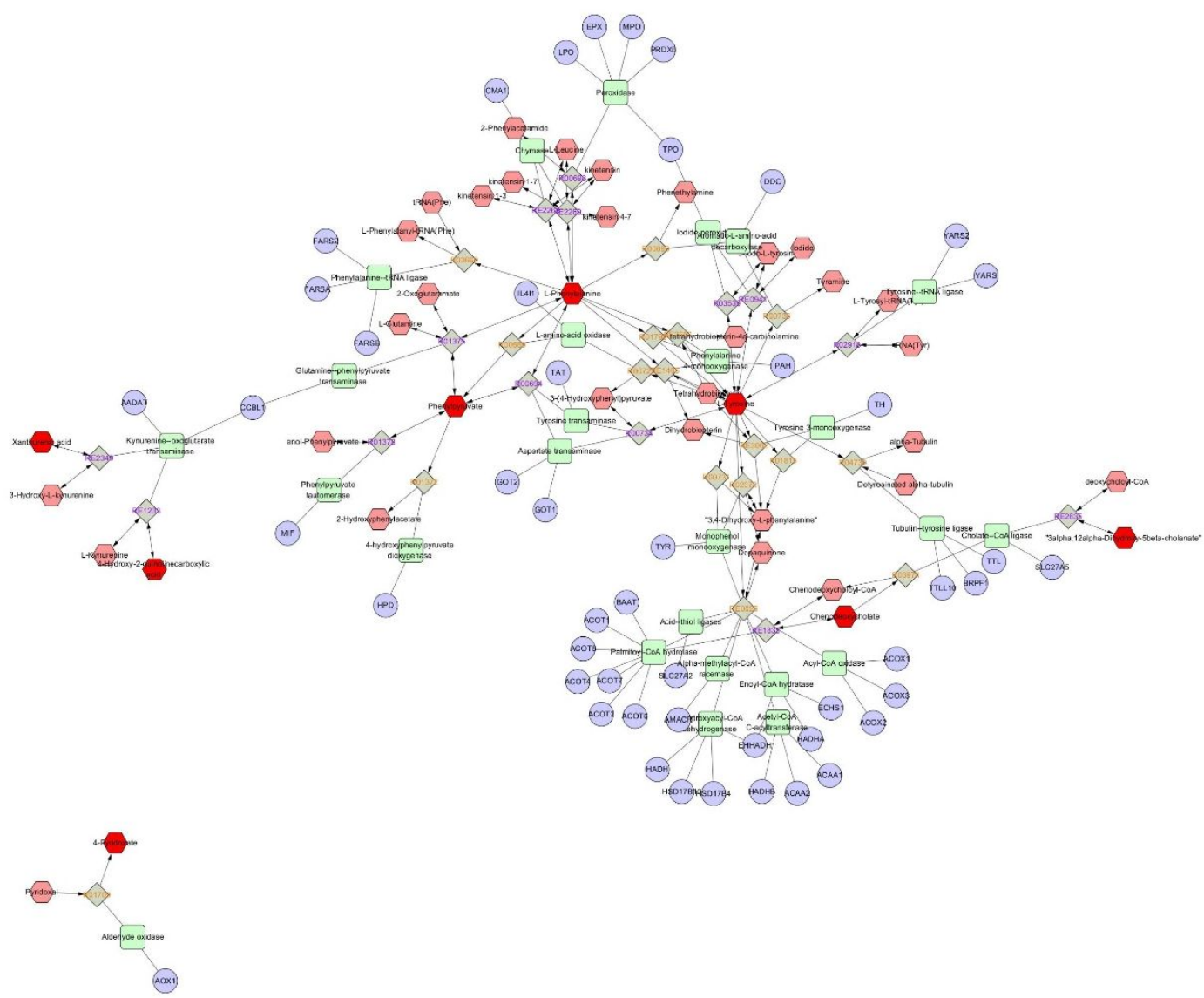

Figure S3. Metabolite-protein targets Correlation

Hexagon : metabolites; round rectangle : enzyme; ellipse $\bigcirc$ Gene; Diamond $\checkmark$ : reaction

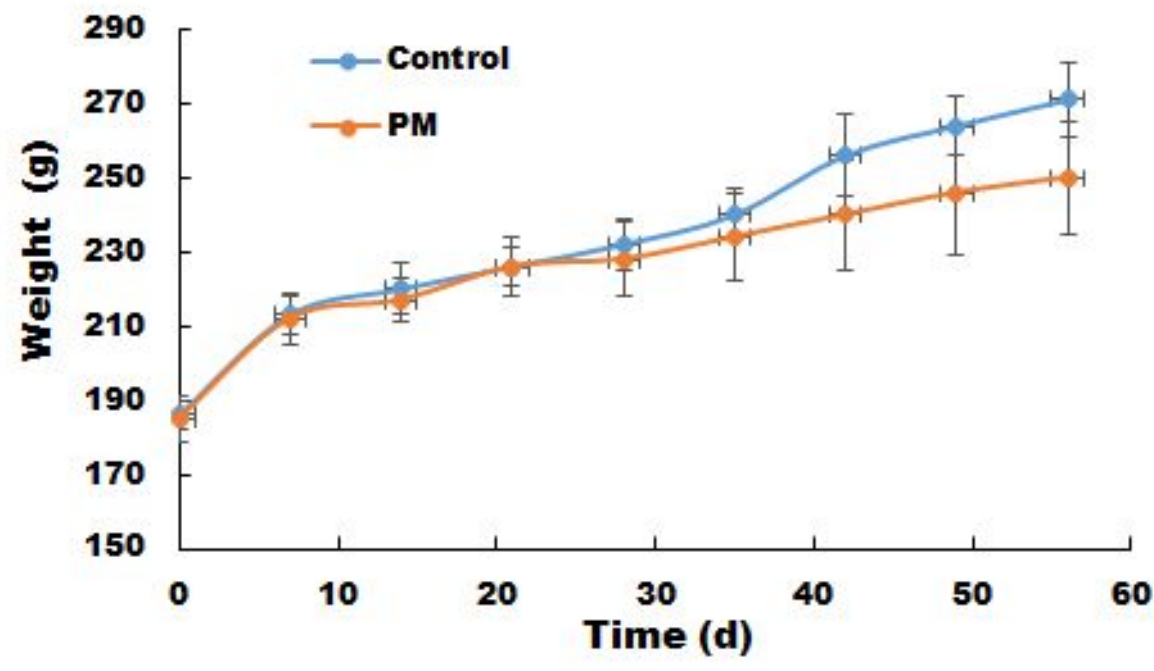

Figure S4 Animals weight $(\mathbf{n}=10)$

During the experiment, the weights of all the animals in PM group were lower than those in the control group, but there was no statistical difference between the two groups 